\title{
ROUGH SETS AND DECISION TABLES
}

\author{
2. Pawlak \\ Institute of Computer Science \\ Polish Academy of Sciences \\ Y.O. Box 22 \\ 00-901 Warsaw, PKiN, Poland
}

\section{Introduction}

we show in this article that the concept of the rough set (see rawlak (1982 and 1985)) can be used as a basis for the decision tables theory (see Pollack, Hicks and Harrison (1971)). The ideas introduced in this paper have been applied to the implementation of cement kiln control algorithm (see Mrózek (1984)) ana showed considerable practical advantages to other methods.

\section{Decision tables}

In this section we give a formal definition of a decision table which will be used throughout this paper.

A decision table is a system

$$
S=\text { (Univ, Att, Val, f) }
$$

where:

$$
\begin{aligned}
& \text { Univ - is a finite set of states, called the universe } \\
& \text { Att }=\text { Con } U \text { Lec - is the set of attributes; Con - is the set of } \\
& \operatorname{VaI}=\bigcup_{a \in A t t} \mathrm{Val}_{a} \text {, where } \mathrm{Val}_{a} \text { is the set of values of an attribute afAtt } \\
& \text { (comain of a). } \\
& f: \text { Univ } \times \text { Att } \rightarrow \text { Val - is a total function, called the decision } \\
& \text { function, such that } f(x, a) \in V_{a l} \text { for every } x \in U n i v \text { and afAtt. }
\end{aligned}
$$
A decision rule in $S$ is a function $\bar{r}_{X}$ : Att $\rightarrow$ Val, such that $f_{x}(a)=$ $f(x, a)$ for every $x \in U n i v$ and a $\in$ Att. 
If $E_{\mathrm{X}}$ is a decision rule in $S$ then $E_{\mathrm{X}} /$ Con and $\mathrm{E}_{\mathrm{X}} /$ Dec are called conditions and decisions of the decision rule $f_{x}$ respectively.

$A$ decision rule $f_{x}$ in $S$ is deterministic (consistent) if for every yeUniv, $y \neq x \hat{I}_{x} /$ Con $=f_{y} /$ Con implies $f_{x} /$ Dec $=f_{y} /$ Dec; otherwise the decision rule $\dot{f}_{x}$ is nondeterministic (inconsistent)。

A decision table $S$ is deterministic (consistent) if all its decision rules are deterministic; otherwise the decision table $s$ is nondeterministic (inconsistent).

A decision table $S^{-}=\left(\mathrm{X}, \mathrm{Att}, \mathrm{Val}{ }^{*}, \mathrm{f}^{*}\right)$ is said to be a $\mathrm{X}$-restriction of the decision table $s=($ Univ, Att, val, $f)$, if $x \subseteq$ Univ, $f^{*}=f / X \times A t t$ and $\operatorname{VaI}=\left\{v \in\right.$ VaI $\left.: \bigvee_{x \in X} f_{x}(a)=v\right\}$

An example of a decision table is shown below:

\begin{tabular}{cccccc} 
Univ & $\mathrm{a}$ & $\mathrm{b}$ & $\mathrm{c}$ & $\mathrm{d}$ & $\mathrm{e}$ \\
\hline 1 & 1 & 0 & 2 & 2 & 0 \\
2 & 0 & 1 & 1 & 1 & 2 \\
3 & 2 & 0 & 0 & 1 & 1 \\
4 & 1 & 1 & 0 & 2 & 2 \\
5 & 1 & 0 & 2 & 0 & 1 \\
6 & 2 & 2 & 0 & 1 & 1 \\
7 & 2 & 1 & 1 & 1 & 2 \\
0 & 0 & 1 & 1 & 0 & 1 \\
& & Fig. & 1 & &
\end{tabular}

In the decision table univ $=\{1,2, \ldots, 8\}$, con $=\{a, b, c\}$, Dec $=\{a, e\}$ ana donains or all attributes are equal $\mathrm{VaI}=\{0,1,2\}$.

3. Rough sets

Let $S=($ Univ,Att, Val,f) be a decision table and let a $\subseteq$ Att, $x, y \in$ Univ,

With every subset of attributes $A \subseteq$ Att we associate the equivalence reiation $\tilde{A}$ defined thus

$$
(x, y) \in \tilde{A} \text { iff } f_{X}(a)=f_{Y}(a) \text { for every } a \in A \text {. }
$$

If $(x, y) \in \widetilde{A}$ we say that $x$ and $y$ are indiscernible with respect to $\widetilde{A}$ in $S(\tilde{A}$ - indiscernible) and $\tilde{A}$ is called an indiscernibility relation in $S$. Equivalence classes of the indiscernibility relation $\tilde{A}$ are called A-elementary sets in $S$ and the family of an equivalence classes of $\widetilde{A}$ is denoted by $\dot{A}^{*}$. 
Let $A \subseteq A t t$ and $X \subseteq$ Univ in a decision table $S=$ luniv, Att, $\operatorname{Val}, f)$.

By A-lower (A-upper) approximation of $X$ in $S$ we mean the sets

$$
\begin{aligned}
& \underline{A X}=\left\{x \in \text { Univ }:[x]_{A} \subset x\right\} \\
& \vec{A} X=\left\{x \in \text { Univ }:[x]_{A} \cap x \neq \phi\right\} .
\end{aligned}
$$

Let $\operatorname{Bn}_{\mathrm{A}}(X)=\overline{\mathrm{A} X}-\underline{\mathrm{AX}}$ will be called $\mathrm{A}$-boundary of $\mathrm{X}$ in $\mathrm{S}$.

we shall use also the following definitions:

$A$ - positive region of set $X$ is the set Ax;

$A$ - doubtful region of set $X$ is the set $B n_{A}(X)$;

$A$ - negative region of set $X$ is the set $\operatorname{Neg}_{A} X=$ Univ - $\bar{A} X$.

If $\overline{A X}=\underline{A X}$ we say that set $X$ is $A$-definable in $S$; otherwise

set $X$ is $A$ - nondefinable in $S$.

Nondefinable sets will be called also rough sets in $\mathrm{S}$.

The number

$$
\alpha_{A}(X)=\frac{\operatorname{card} \underline{A X}}{\operatorname{card} \bar{A} X}
$$

will be called the accuracy of the $X$ with respect to $A$ in $S$, and the number

$$
\rho_{A}(X)=1-\alpha_{A}(X)
$$

will be called the roughness of the set $X$ with respect to $A$ in $S$.

Let us notice that each subset of attributes $A \subseteq$ Att in a decision table $S=(U n i v, A t t, V a l, E)$ defines uniquelly the topological space $T_{S}=$ (Univ, Def $\left.f_{A}(S)\right)$, where $\operatorname{Def}_{A}(S)$ is the family of all $A$ definable sets in $S$, and the lower and upper approximations are interior and closure in the topological space $\mathrm{T}_{\mathrm{S}}$, thus approximations have the following properties:

1) $\underline{\mathrm{AX}} \subseteq \mathrm{X} \subseteq \overline{\mathrm{A} X}$

2) $\underline{\not} \phi=\bar{A} \phi=\varnothing ; \underline{A}$ Univ $=\bar{A}$ Univ $=$ Univ

3) $\underline{A}(X \cup Y) \supseteq \underline{A} \cup \underline{A}$

4) $\overline{\bar{A}}(X \cup Y)=\overline{\bar{A} X} \cup \overline{\bar{A}} Y$

5) $\underline{A}(X \cap Y)=A X \cap \underline{A X}$

6) $\overline{\bar{A}}(X \cap Y) \subseteq \overline{\bar{A}} X \cap \overline{\bar{A}} Y$

7) $A(-X)=-\bar{A}(X)$

8) $\overline{\bar{A}}(-X)=-\underline{A}(X)$

Moreover in this topological space we have the following two properties: 
9) $\begin{aligned} \overline{A A X} & =\bar{A} \bar{A} X \\ \bar{A} \bar{A} X & =\bar{A} \overline{\bar{A}} X\end{aligned}$

itrom the topological view the rough sets can be classified as follows:
a) Set $X$ is roughly $A$-definable in $S$ if $\underline{A X} \neq \varnothing$ and $\bar{A} X \neq$ Univ,
b) Set $X$ is internally A-nondefinable in $S$ if $\underline{A X}=\varnothing$ and $\bar{A} X \neq$ Univ,
c) Set $X$ is externally $A$-nondefinable in $S$ if $\bar{A} X=$ Univ and $\underline{A} \neq \neq$,
d) Set $X$ is totally $A$-nondefinable in $S$ if $\underline{A X}=\not D$ and $\overline{A X}=$ Univ.

\section{Dependency of attributes}

Let $s=($ univ, $\mathrm{ktt}, \mathrm{Val}, \mathrm{f})$ be a decision table, $E=\left\{\mathrm{x}_{1}, \mathrm{x}_{2}, \ldots, \mathrm{x}_{\mathrm{n}}\right\}$, where $x_{i} \subseteq$ Univ, a family of subsets of Univ and $A \subseteq$ Att.

By A-lower (A-upper) approximation of $F$ in $S$ we mean the families

$$
\begin{aligned}
& \underline{\mathrm{AF}}=\left\{\mathrm{AX}_{1}, \overline{\mathrm{AX}}_{2}, \ldots, \overline{\left.\mathrm{AX}_{n}\right\}}\right. \\
& \overline{\mathrm{A}} \mathrm{F}=\left\{\overline{\mathrm{AX}}_{1}, \overline{\mathrm{A}} \mathrm{X}_{2}, \ldots, \overline{\mathrm{A} \mathrm{X}_{\mathrm{n}}}\right\} .
\end{aligned}
$$

whe $A$-positive region of a family $F$ is the set

$$
\operatorname{Pos}_{A}(\vec{F})=\bigcup_{X_{i} \in F} \underline{A X}_{i} .
$$

The A-doubtful region of a family $F$ is the set

$$
B n_{A}(F)=\bigcup_{X_{i} \in F} B n_{A} X_{i} .
$$

The is - negative region of a family $F$ is the set

The numoer

$$
\operatorname{Neg}_{A}\left(\bar{r}^{\prime}\right)=\operatorname{Univ}-\bigcup_{X_{i} \in F} \bar{A} X_{i} .
$$

$$
\Upsilon_{\mathrm{A}}(F)=\frac{\operatorname{card} \operatorname{Pos}_{\mathrm{A}}(E)}{\operatorname{card} U}
$$

will be called the quality at the approximation of $F$ by $A$ in $S$, and the number

$$
B_{A}(F)=\frac{\operatorname{card} \operatorname{Pos}_{A}(F)}{\sum_{X_{i} \in F} \operatorname{card} \bar{A} X_{i}}
$$


will be called the accuracy at approximation of $F$ by $A$ in $S$.

Let $B, C \subseteq A t t$ be two subsets of attributes in $S=$ (Univ, Att, Val,fl, and $k$ real number such that $0 \leqslant k \leqslant 1$.

we say that $C$ depends in a degree $k$ on $B$ in $S$, in symbols $B \rightarrow C$, if $k=Y_{B}\left(C^{*}\right)$.

If $k=1$ we say that $C$ totally depends on $B$ in $S$ and we write also $B \rightarrow C$ instead of $B \rightarrow C$.

If $0<k<1$ we say that $C$ roughly aepends on $B$ in $S$.

If $k=0$ we say that $C$ is totally independent on $B$ in $s$.

The following properties are valid:

Property 1

A aecision table $S=($ Univ, Att, Val,f) is deterministic iff Con $\rightarrow$ Dec in $s$.

A decision table $S=$ (Univ, Att, Val, $f$ ) is called roughly deterministic if con $\stackrel{k}{\rightarrow}$ Dec and $0<k<1$.

I decision table $S=$ (Univ,Att, Val, f) is called totally nondeterministic if Con $\stackrel{0}{\rightarrow}$ Dec.

Property 2

The following properties are true:

$1)$ Con $\rightarrow$ Dec in S/Pos Con $\left(\right.$ Dec $\left.^{*}\right)$

2) Con $\stackrel{0}{\rightarrow}$ Dec in $S / \mathrm{Bn}_{\mathrm{Con}}\left(\right.$ Dec $\left.^{*}\right)$.

Note. The above property says that every decision table can be decomposed into two parts (possibly empty) such that one is deterministic and the second totally nondeterministic.

It is easy to compute that in the decision table shown in Fig. 1, Los $_{\mathrm{Con}}\left(\mathrm{Dec}^{*}\right)=\{3,4,6,7\},, \quad \mathrm{Bn}_{\mathrm{Con}}\left(\mathrm{DeC}^{*}\right)=\{1,2,5,8\}$, $\gamma_{\text {Con }}\left(\right.$ Dec $\left.^{*}\right)=0.5$, i.e. Con $\stackrel{0,5}{\rightarrow}$ Dec, that is to mean that the decision table is roughiy deterministic and can be decomposed into the following two decision tables

\begin{tabular}{cccccc} 
Univ & $a$ & $b$ & $c$ & $d$ & $e$ \\
\hline 3 & 2 & 0 & 0 & 1 & 1 \\
4 & 1 & 1 & 0 & 2 & 2 \\
6 & 2 & 2 & 0 & 1 & 1 \\
7 & 2 & 1 & 1 & 1 & 2 \\
& & Fig。 & 2 & &
\end{tabular}




\begin{tabular}{cccccc} 
Univ & $\mathrm{a}$ & $\mathrm{b}$ & $\mathrm{c}$ & $\mathrm{d}$ & $\mathrm{e}$ \\
\hline 1 & 1 & 0 & 2 & 1 & 0 \\
2 & 0 & 1 & 1 & 1 & 2 \\
5 & 1 & 0 & 2 & 0 & 1 \\
8 & 0 & 1 & 1 & 0 & 1 \\
& Fig. & 3 & &
\end{tabular}

The decision table shown in Fig. 2 is totally deterministic and the table show in Fig. 3 is totally nondeterministic,

Let us also notice that $B_{\text {Con }}\left(D^{*}{ }^{*}\right)=1 / 3$ which is the ratio of all deterministic decisions to all possible decisions in the table. The meaning of the number ${ }^{\beta}$ Con $\left(\right.$ Dec $\left.{ }^{*}\right)$ is obvious.

\section{Reduction of attributes}

Let $S=($ Univ, Att, Val,f) be a decisjon table and let $A \subseteq$ Att.

Set $A$ is independent in $S$ if for every $B \subset A, \widetilde{B} \supset \widetilde{A}$.

Set $A$ is dependent in $S$ if there exists $B \subset A$ such that $\widetilde{B}=\tilde{A}$.

Set $B \subseteq A$ is a reauct of $A$ in $S$ if $B$ is the maximal indepenaent set in $S$.

Subset $B \subseteq A$ is a reduct of $A$ with respect to $C \subseteq A$ t in $S$ if $B$ is an independent subset of $A$ such that $\gamma_{B}\left(C^{*}\right)=\gamma_{A}\left(C^{*}\right)$ for $\left.\operatorname{Pos}_{B}\left(C^{*}\right)=\operatorname{pos}_{A}\left(C^{*}\right)\right)$.

Let us notice that if $A=C$ the reduct of $A$ with respect to $C$ coincide with the reduct of $A$ 。

Property 3

If $A \stackrel{K}{\rightarrow} B$ in $S$ and $C$ is a reduct of $A$, or reduct of $A$ with respect to $B$ in $S$, then $C \stackrel{k}{\rightarrow}$,

In particular if $C$ is a reduct of conditions attributes con in a decision table $s$ and $C o n \stackrel{k}{\rightarrow}$ Dec, then $c \stackrel{k}{\rightarrow}$ Dec. This is to mean that we can simplify the decision table by reducing the set of conditions attributes.

For example in the decision table shown in Fig. 1 the only reduct of conditions attributes is $c=\{a, b\}$, thus we can simplify the decision table as shown below: 


\begin{tabular}{ccccc} 
Univ & $a$ & $b$ & $d$ & $e$ \\
\hline 1 & 1 & 0 & 2 & 0 \\
2 & 0 & 1 & 1 & 2 \\
3 & 2 & 0 & 1 & 1 \\
4 & 1 & 1 & 2 & 2 \\
5 & 1 & 0 & 0 & 1 \\
6 & 2 & 2 & 1 & 0 \\
7 & 2 & 1 & 1 & 2 \\
8 & 0 & 1 & 0 & 1 \\
& Fig. & &
\end{tabular}

and consequently the decision table can be decomposed as follows

\begin{tabular}{ccccc} 
Univ & $a$ & $b$ & $d$ & $e$ \\
\hline 3 & 2 & 0 & 1 & 1 \\
4 & 1 & 1 & 2 & 2 \\
6 & 2 & 2 & 1 & 1 \\
7 & 2 & 1 & 1 & 2 \\
& Fig. & 5 \\
Univ & $a$ & $b$ & $d$ & $e$ \\
\hline 1 & 1 & 0 & 2 & 0 \\
2 & 0 & 1 & 1 & 2 \\
5 & 1 & 0 & 0 & 1 \\
8 & 0 & 1 & 0 & 1 \\
& Fig. & 6 & &
\end{tabular}

It can be easily seen that the set of decision attributes in the table is independent.

We can also define the approximate reduct (or approximate reduct with respect to a subset $C$ ) in the following way:

Let $0 \leqslant \varepsilon \leqslant 1$ be a real number and let $B \subseteq A \subseteq$ Att in a decision table $S=($ Univ, Att, Val, f).

Subset $B$ of $A$ is a $\varepsilon$-reduct of $A$ in $S$ if $B$ is independent in $S$ and $\Upsilon_{B}\left(A^{*}\right)=1-\varepsilon$.

Subset $B$ of $A$ is a $\varepsilon$-reduct of $A$ in $S$ with respect to $C \subseteq$ Att if $B$ is inäependent in $S$ and $\gamma_{B}\left(C^{*}\right)=\gamma_{A}\left(C^{*}\right)=\varepsilon$ 。

Directly from these definitions we have

\section{Property 4}

If $B$ is a $\varepsilon$-reduct of $A$ in $S$ then $B \stackrel{1-\varepsilon}{\longrightarrow} A$. 


\section{property 5}

If $B$ is a $\varepsilon$-reduct of $A$ in $S$ with respect to $C \subseteq A t t$, ana $A \stackrel{k}{\rightarrow} C$, then $B \stackrel{k-\varepsilon}{\longrightarrow} C$.

In particular if Con $\frac{k}{\rightarrow}$ Dec in $S$ and $C \subseteq C$ is a $\varepsilon$-reduct of Con in $S$, then $C \stackrel{k-\varepsilon}{\longrightarrow}$ Dec. That is to mean that we can reduce the set of conditions attributes, in such a way that the degree of dependency between decisions and conditions attributes is decreased by the constant $\varepsilon$.

\section{Cost of decision table}

With every decision table $S=$ (Univ,Att, Val,f) and subset of conditions attributes $B \subseteq$ Con, we associate cost $C_{S, B}$ or in short $C_{B}$, when $s$ is understood defined thus:

$$
C_{B}=\sum_{a \in B} C_{1}(a)+\sum_{x \in B n_{B}}^{\Sigma}\left(D_{e C}^{*}\right) c_{2}(x),
$$

where

$$
\mathrm{C}_{1}: \operatorname{Con} \rightarrow \mathrm{R}^{+}, \quad \mathrm{C}_{2}: \text { Univ } \rightarrow \mathrm{R}^{+}
$$

and $\mathrm{K}^{+}$is the set of nonnegative reals; $c_{1}(a)$ - is the cost of measuring the value of attribute a and $c_{2}(x)$ - is the cost of imprecise classification of $x$, due to smallex set of attributes (imprecise decision in state $x$ ).

There is of course trade-off between $c_{1}$ and $c_{2}$ and we can minimize the total cost $C_{B}$ by proper choosing of conditions attributes.

The problem will be discussed in some details in a subsequent paper.

\section{Decision nets}

viany problems require not one decision table, but a set of different decision tables connected in a net in such a way that if some conditions are satisfied, the decision making process can be switched from one decision table to another one.

In order to do so assume that each decision table is labelled by a "name" of the decision table, and the set of decision attributes in each decision table contains one specific attribute - values of which are names of decision tables. Thus each condition in the decision table 
specifies also next decision table to be used, to make a decision.

With each set of such decision tables we can associate a directed graph, nodes of which are labelled by decision tables, and are labellea by conditions, which determine transfers between corresponding tables.

For example consider three decision tables $A, B, C$ as shown in Eig. 7 .

A

\begin{tabular}{|c|c|}
\hline $\mathrm{a}$ & $b$ \\
\hline
\end{tabular}

$\begin{array}{lllll}1 & 0 & 1 & 2 & \text { A }\end{array}$

$\begin{array}{lllll}2 & 2 & 1 & 1 & B\end{array}$

$\begin{array}{lllll}3 & 0 & 0 & 0 & \text { 蕅 }\end{array}$

$\begin{array}{lllll}4 & 1 & 1 & 2 & C\end{array}$
B

\begin{tabular}{|c|c|c|c|c|c|c|c|c|c|}
\hline$u$ & $\mathrm{a}$ & $b$ & $c$ & $\mathbf{N}$ & $u$ & a & b & $\mathrm{C}$ & $\mathbb{N}$ \\
\hline 1 & 1 & 2 & 2 & B & 1 & 0 & 2 & 2 & C \\
\hline 2 & 0 & 0 & 0 & $\mathrm{~A}$ & 2 & 1 & 0 & 1 & $\mathrm{C}$ \\
\hline 3 & 2 & 1 & 1 & $\mathbb{A}$ & 3 & 2 & 1 & 0 & A \\
\hline 4 & 2 & 0 & 2 & B & & & & & \\
\hline 5 & 1 & 2 & 1 & $C$ & & & & & \\
\hline
\end{tabular}

Fig. 7

$\mathrm{N}$ - is the "next table" attribute.

The graph associated with this set at tables is shown in Fig. 8 .

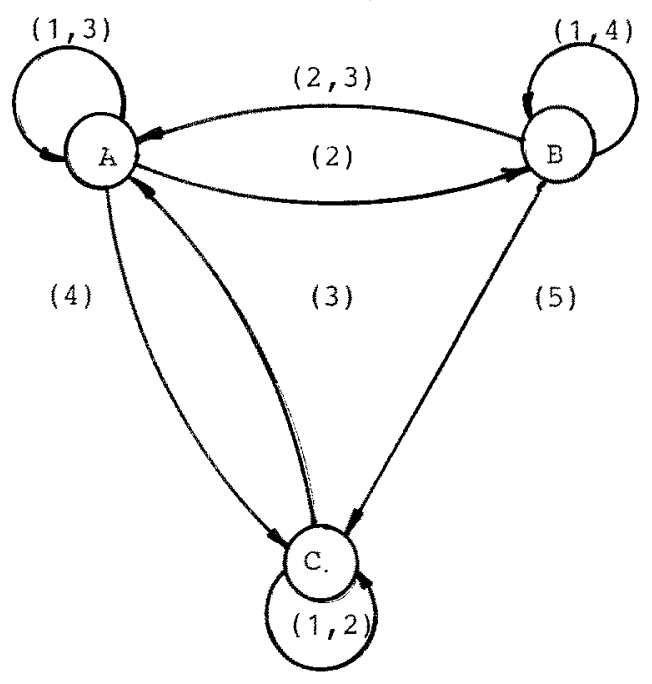

Eig. 8

For the sake of simplicity we labelled branches of the graph not by conaitions but corresponding states.

Several theoretical problems arise in connection with decision nets, for example the problem of consistency (the decision not shown in $\mathrm{Fig} .8$ is inconsistent!) but we shall not discuss these problems 
in these paper.

Acknowledgement. Thanks are due to dr. A. Skowron for critical remarks.

\section{References}

irózek, A., (1984). Information Systems and Control Algorithms, Bull. polish Acad. Sci. (to appear)

fawlak, Zo, (1982). Rough Sets. International Journal of Information and Computer Sciences, 11(5), 341-356

Fawlak, $Z_{0},(1985)$. Decision tables and decision algorithms. Bull. Folisin Acad. Sci. (to appear)

rollack, S., Hicks, H., and Harrison, W., (1971)。 Decision Tables: Theory and Practice. Wiley and Sons, Inc. New York. 